\title{
Ferromagnetic Domain Behaviors in Mn doped ZnO Film
}

\author{
Devaraj Soundararajan', Jaime Santoyo-Salazar ${ }^{2}$, Jang-Myoun $\mathrm{Ko}^{3 *}$, and Ki Hyeon $\mathrm{Kim}^{1 *}$ \\ ${ }^{1}$ Department of Physics, Yeungnam University, Gyeongsan 712-749, Korea \\ ${ }^{2}$ Centro de Investigación y de Estudios Avanzados de Instituto Politécnico Nacional, Departamento de Física, Av. IPN \\ 2508, San Pedro Zacatenco, C.P. 07360, A.P. 14-740, 07000 México, D.F., Mexico \\ ${ }^{3}$ Division of Applied Chemistry and Biotechnology, Hanbat National University, Daejeon 305-719, Korea
}

(Received 18 August 2011, Received in final form 5 September 2011, Accepted 15 September 2011)

\begin{abstract}
Mn doped $\mathrm{ZnO}$ films were prepared on $\mathrm{Si}(100)$ substrates using sol-gel method. The prepared films were annealed at $550^{\circ} \mathrm{C}$ for decomposition and oxidation of the precursors. XRD analysis revealed the presence of $\mathrm{ZnMnO}$ hexagonal wurtzite phase along with the presence of small quantity of $\mathrm{ZnMn}_{2} \mathrm{O}_{3}$ secondary phase and poor crystalline nature. The 2D, 3D views of magnetic domains and domain profiles were obtained using magnetic force microscopy at room temperature. Rectangular shaped domains with an average size of $4.16 \mathrm{~nm}$ were observed. Magnetic moment measurement as a function of magnetic field was measured using superconducting quantum interference device (SQUID) magnetometry at room temperature. The result showed the ferromagnetic hysteresis loop with a curie temperature higher than $300 \mathrm{~K}$.
\end{abstract}

Keywords : Mn doped $\mathrm{ZnO}$ film, sol gel method, magnetic domains, M-H plot

\section{Introduction}

Materials with novel combinations of properties such as room temperature ferromagnetism and semiconducting properties are required for spintronic devices. Dilute Magnetic Semiconductors (DMS) in which magnetic ions substitute cations of the host semiconducting material are assumed to be ideal systems for spintronics [1-3]. DMSs based on II-VI and III-V compound semiconductors have attracted much attention in recent years because they provide material foundation for semiconductor spintronic devices. The discovery of ferromagnetism in (In, Mn)As and $(\mathrm{Ga}, \mathrm{Mn}) \mathrm{As}$ prepared by MBE method promoted DMSs as fundamental materials for spintronics because of their compatibility with semiconductors used in present day electronics [4]. Likewise, ferromagnetism in $(\mathrm{Zn}$, Cr)Te prepared by MBE technique [5] has also stimulated the research in this field. In our recent study, we have investigated ferromagnetism and its origin in $(\mathrm{Zn}, \mathrm{Cr}) \mathrm{Te}$ films prepared by a simple thermal evaporator $[6,7]$. All these reports mainly put forward the below room temperature ferromagnetic behavior and the ferromagnetic cluster within magnetic semiconductors as the bane of semiconductor spintronics in order to apply effectively for practical applications [8]. Therefore, the research on different preparation routes, semiconducting hosts and magnetic impurites are employed to achieve the desired goals.

In this manner, transition metal oxide based DMSs have been attracting much attention, due to their important optoelectronic properties and potential applications in spintronics at practical temperatures [9-12]. $\mathrm{ZnO}$ is one among them posing great interest in science and technology due to its versatile features suitable for a variety of applications. A large number of reports are available on the magnetic properties of transition metal doped $\mathrm{ZnO}$ [13-15]. Sharma et al. [16] have published the first observation of room temperature ferromagnetism in $\mathrm{Mn}$ doped $\mathrm{ZnO}$. Ferromagnetism near or above room temperature has already been reported for Mn-alloyed $\mathrm{ZnO}$ nanocrystals, pellets and thin films $[17,18]$. In the present study, we demonstrate the magnetic properties such as magnetic domain observation and magnetic moment measurements for the $\mathrm{Mn}$ doped $\mathrm{ZnO}$ film grown on $\mathrm{Si}$ (100) using sol-gel technique.

\footnotetext{
*Corresponding author: Tel: +82-53-810-2334

Fax: +82-53-810-4616, e-mail: kee1@ynu.ac.kr
} 


\section{Experimental}

\subsection{Preparation}

For the preparation of $\mathrm{ZnO}$ and $\mathrm{Mn}$ doped $\mathrm{ZnO}$ films, the precursor materials used were zinc acetate dihydrate, isopropyl alcohol, diethanolamine and manganese chloride tetra hydrate. Total concentration of the metal ions was $0.1 \mathrm{M}$. A sol was prepared by mixing thoroughly the calculated amount of the corresponding salts in isopropyl alcohol at room temperature. When it turned milky an equimolar quantity of diethanolamine was added as a sol stabilizer drop by drop into the solution until it becomes transparent. Dip coating method was used to coat the silicon (100) substrate after proper mixing of the sol. Totally 10 dipping were carried out. After each dipping (coating), the film was dried at $110^{\circ} \mathrm{C}$ in hot air oven for 15 minutes. Finally, the films were annealed for 1 hour in muffle furnace at $550^{\circ} \mathrm{C}$ for decomposition and oxidation of the precursors.

\subsection{Characterization}

In the present study, crystalline structure of the prepared films was analyzed using X-ray diffractometer [ModelShimadzu (XRD-6000), $\lambda=1.5406 \AA$ ] operated in the $2 \theta$ scan range of $20^{\circ}$ to $80^{\circ}$. Ex-situ topography and surface magnetic domain pattern study was performed at room temperature by means of Scanning Probe Microscope (SPM) (JEOL JSPM-4210) to scan particle size and magnetic domains at room temperature. Topography of the films was scanned by Tapping ${ }^{\mathrm{TM}}$ mode and the magnetic domains were analyzed by lift mode. A magnetic cantilever tip NSC14/Co-Cr, Mikro-masch, Co. was used with resonant frequency of $160 \mathrm{kHz}$. The magnetic domains were observed in lift mode MFM with interaction in the height of $25 \mathrm{~nm}$ and output of $0.025 \mathrm{Amp} / \mathrm{V}$. The 2D, 3D images, profiles, and domain measurements were processed with the software win SPM DPS, JEOL Ltd. Magnetic moment versus magnetic field was measured by using a SQUID magnetometry (Quantum Design MPMS-XL Superconducting Magnet) at $300 \mathrm{~K}$.

\section{Results and Discussion}

\subsection{Structure}

$\mathrm{X}$-ray diffraction patterns of the $\mathrm{ZnO}$ and the $\mathrm{Mn}$ doped $\mathrm{ZnO}$ films are shown in Fig. 1. The pure $\mathrm{ZnO}$ shows amorphous nature without any significant peaks. However, the $\mathrm{Mn}$ doped $\mathrm{ZnO}$ film exhibits poor crystallanity along with the $\mathrm{ZnMnO}$ hexagonal wurtzite phase. Peaks at scattering angles $(2 \theta)$ of $32.05^{\circ}, 34.45^{\circ}, 36.53^{\circ}$ and $62^{\circ}$ correspond to reflections from (100), (002), (101) and

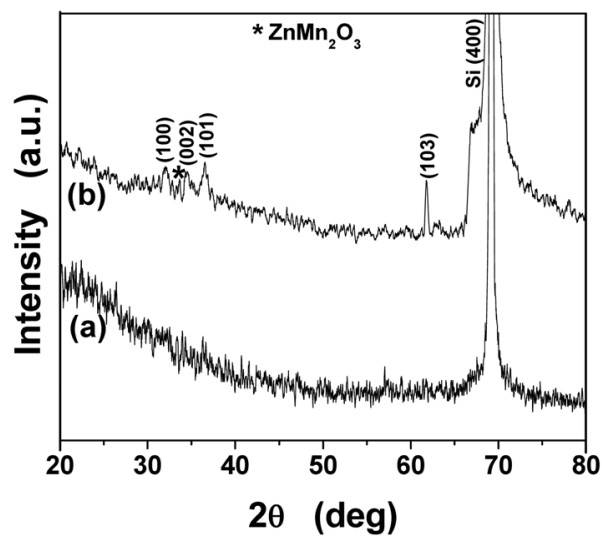

Fig. 1. X-ray diffraction patterns of (a) $\mathrm{ZnO}$ and (b) Mn doped $\mathrm{ZnO}$ films deposited on $\mathrm{Si}$ (100).

(103) crystal planes, respectively. These reflections confirm the existence of $\mathrm{ZnMnO}$ hexagonal wurtzite phase [19]. Also, a tiny peak at around $33^{\circ}$ labeled by a star mark indicates the presence of metastable $\mathrm{ZnMn}_{2} \mathrm{O}_{3}$ like secondary phase [20]. This shows the solubility of $\mathrm{Mn}$ in $\mathrm{ZnO}$ host is moderate and so the Mn distribution is not much efficiently uniform.

\subsection{Ferromagnetic domains}

Magnetic force microscopy detects the force derivative acting on a small magnetic probe in the stray field close to the specimen. The technique can give a resolution below $20 \mathrm{~nm}$ with comparably low effort in sample preparation [21]. MFM imaging can therefore be used to sensitively probe micro magnetism of thin film samples. The purpose of this section is to demonstrate the application of MFM to investigate magnetic characteristics of domains as well as stray fields in individual grains of the film.

Prior to MFM analysis, the film was exposed to argon gas blow and consequently the topographic scanning was made by AFM in tapping mode. The topography and magnetic domains of the $\mathrm{ZnMnO}$ film grown on the $\mathrm{Si}$
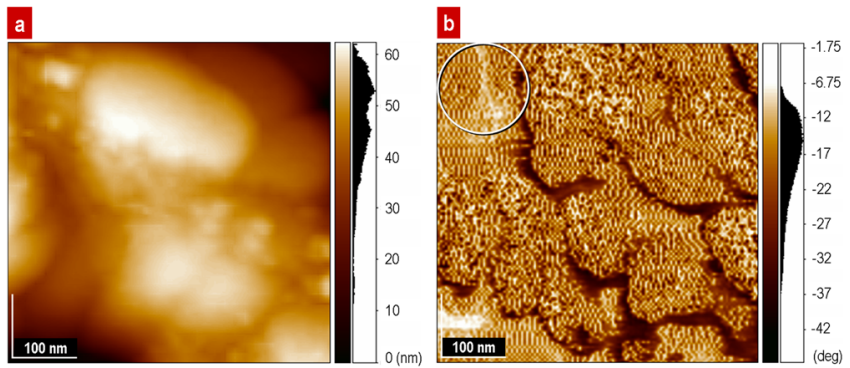

Fig. 2. (Color online) (a) AFM and (b) MFM images of Mn doped $\mathrm{ZnO}$ film grown on $\mathrm{Si}(100)$. 
(100) substrate is shown in Fig. 2. The AFM image in the scanned area of $500 \mathrm{~nm} \times 500 \mathrm{~nm}$ shows the nanoparticles of $\mathrm{ZnMnO}$. To the right of the AFM image indicates profile of a roughness in average of $60 \mathrm{~nm}$. The $\mathrm{ZnMnO}$ films have submicrometric grains in the order of $150 \mathrm{~nm}$. The MFM image scanned in the same area shows multiple domains within individual grains (domain islands). Also, the overlapping of islands can be seen owing to the multiple dipping procedures carried out for the film deposition. Within each island where the magnetic interactions are observed as nanometric rectangular shapes. The stripes inside of each island contain magnetic domains which have different positions due to different magnetic force caused within islands. It results in the anisotropic behavior of domains in the sample surface. The circle in the center of figure indicates the selected area for the MFM analysis and to the right of the profile indicates the lift high of $15^{\circ}$.

The marked region indicated in Fig. 2(b) is taken in order to analyze the magnetic domains in the islands as shown in Fig. 3(a). This high resolution image shows more explicitly the rectangular shaped domains. The domains are isotropic within individual grains (domain
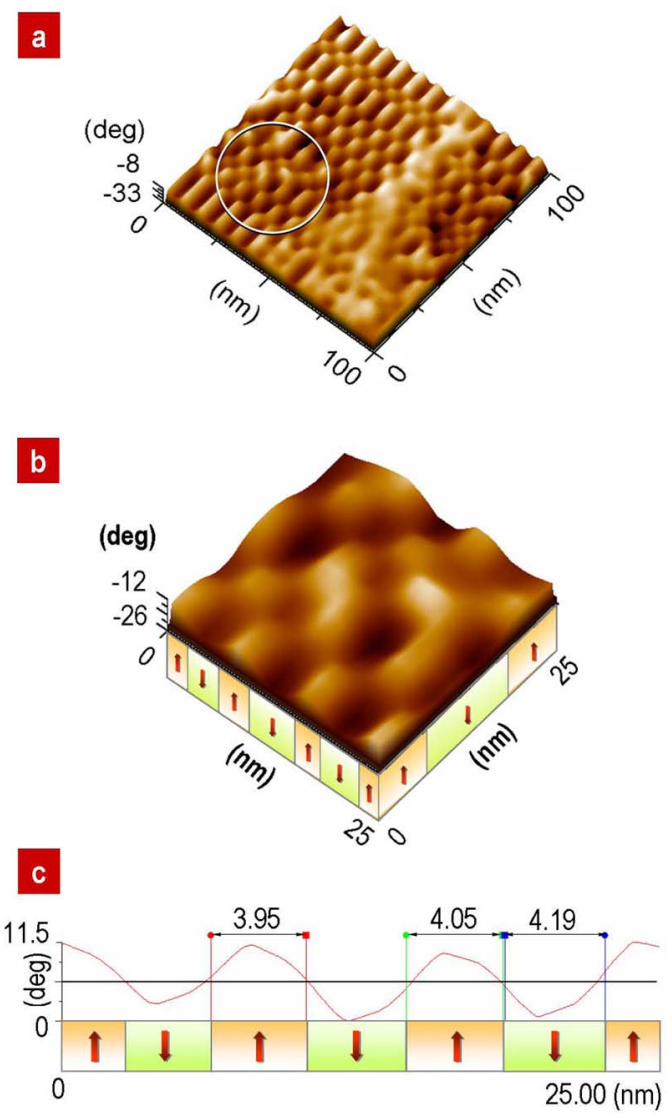

Fig. 3. (Color online) (a) 2D (b) 3D (c) profile images of magnetic domains. islands). Further, domains in 3D view are observed in the marked region $(25 \mathrm{~nm} \times 25 \mathrm{~nm})$ as shown in Fig. 3(b). It clearly shows the domains as bright and dark contrast. The bright and dark contrast represents domains with positive $(\uparrow)$ and negative $(\downarrow)$ polarizations, respectively. Bottom of Fig. 3(b) shows cross sectional view of the domains. The distribution of domains in the profile in Fig. $3(c)$ indicates domains with an average size of $4.16 \mathrm{~nm}$. All these results ensure the observation of domains at room temperature, which implies the existence of magnetization at the surface of the film.

\subsection{M-H curve}

In addition to the surface sensitive MFM technique to observe magnetic domains, we also used the bulk sensitive SQUID magnetometer to measure magnetic moment versus field plot $(\mathrm{M}-\mathrm{H})$ at room temperature and the result is shown in Fig. 4. It obviously shows hysteresis loop. Since the diamagnetic contribution from the Si (100) substrate was not subtracted, its influence on the magnetic behavior of the film was seen. The inset shows the asobtained M-H plot. The behavior of the as-obtained M-H curve clearly shows diamagnetic contribution from the $\mathrm{Si}$ substrate and it dominates net magnetic moment from the film in the high field region. The expanded view of the plot shows a clear hysteresis curve indicating net ferromagnetic behavior from the $\mathrm{ZnMnO}$ film.

However, it is not yet clear whether the observed ferromagnetic behavior is truly intrinsic or related to secondary phases such as ferromagnetic clusters. Though the origin of ferromagnetism in $\mathrm{Mn}$ doped $\mathrm{ZnO}$ is still in debate, it can be partly interpreted by using XRD pattern. Poor crystallinity of the $\mathrm{ZnMnO}$ film shows Mn incorporation and distribution is not effective. Therefore, un-

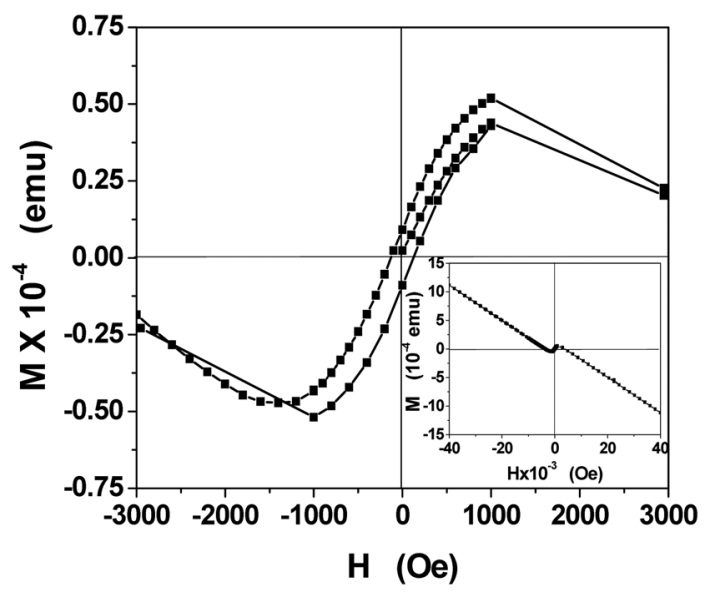

Fig. 4. Magnetic moment measurements as a function of magnetic field on $\mathrm{ZnMnO} / \mathrm{Si}(100)$ at $300 \mathrm{~K}$. Inset shows the asobtained plot. 
detectable precipitates of $\mathrm{Mn}$ and its oxides such as $\mathrm{MnO}$, $\mathrm{MnO}_{2}, \mathrm{Mn}_{2} \mathrm{O}_{3}$ and $\mathrm{Mn}_{2} \mathrm{O}_{4}$ are also possible in the film, in addition to the existence of the $\mathrm{ZnMnO}$ wurtzite phase and the tiny $\mathrm{ZnMn}_{2} \mathrm{O}_{3}$ related intermediate phase. $\mathrm{Mn}$, $\mathrm{MnO}$ and $\mathrm{MnO}_{2}$ are antiferromagnetic with Neel temperatures of $116 \mathrm{~K}$ and $84 \mathrm{~K}$, respectively [20, 22]. $\mathrm{Mn}_{3} \mathrm{O}_{4}$ is ferromagnetic ordering, and its Curie temperature is around $44 \mathrm{~K}$ [23]. It is already reported that the curie temperature of $\mathrm{ZnMnO}$ and $\mathrm{ZnMn}_{2} \mathrm{O}_{3}$ like phase are well above $300 \mathrm{~K}$ $[20,24,25]$. Therefore, the obtained results from XRD suggest that the dominating $\mathrm{ZnMnO}$ wurtzite phase and minute $\mathrm{ZnMn}_{2} \mathrm{O}_{3}$ related secondary phase should play a major role for the observed room temperature ferromagnetism from the $\mathrm{Mn}$ doped $\mathrm{ZnO}$ film. The observation of surface and bulk magnetic properties have shows clearly the room temperature ferromagnetic behavior and hence the Curie temperature is expected to be greater than $300 \mathrm{~K}$.

\section{Conclusion}

Mn doped $\mathrm{ZnO}$ films were grown on $\mathrm{Si}$ (100) substrate using sol-gel method. XRD revealed poor crystallinity with $\mathrm{ZnMnO}$ hexagonal wurtzite phase and a weak signal of metastable $\mathrm{ZnMn}_{2} \mathrm{O}_{3}$ secondary phase. AFM image showed sub nanometric particles with an average size of $145 \mathrm{~nm}$. MFM images clearly showed the presence of rectangular shaped multiple domains with an average size of $4.16 \mathrm{~nm}$ in the particles. M-H measurement showed a ferromagnetic hysteresis behavior even at $300 \mathrm{~K}$, suggesting high Curie temperature of the prepared $\mathrm{Mn}$ doped $\mathrm{ZnO}$ film.

\section{References}

[1] F. V. Kyrychenko and J. Kossut, Physica E. 10, 378 (2001).

[2] K. Takabayashi, N. Takahashi, I. Yagi, K. Yui, I. Souma, J. X. Shen, and Y. Oka, J. Lumin. 87, 347 (2000).

[3] L. Parthief, S. Luthef, O. Portugall, M. von Ortenberg, K. Uchidab, H. Kunimatsub, and N. Miurab, J. Cryst. Growth 184, 339 (1998).

[4] H. Ohno, A. Shen, F. Matsukura, A. Oiwa, A. Endo, S. Katsumoto, and Y. Iye, App. Phys. Lett. 69, 363 (1996).

[5] H. Saito, S. Yamagata, and K. Ando, J. Appl. Phys. 95, 7175 (2004).

[6] D. Soundararajan, D. Mangalaraj, D. Nataraj, L. Dorosinskii, J. Santoyo-Salazar, K. Senthil, and J. M. Ko, Sci. Adv. Mater. 3, 1 (2011).

[7] D. Soundararajan, D. Mangalaraj, D. Nataraj, L. Dorosin- skii, J. Santoyo-Salazar, and M. J. Riley, J. Magn. Magn. Mater. 321, 4108 (2009).

[8] P. T. Chiu and B. W. Wessels, Appl. Phys. Lett. 90, 207202 (2003).

[9] K. Ueda, H. Tabata, and T. Kawai, Appl. Phys. Lett. 79, 988 (2001).

[10] T. Fukumura, Z. Jin, M. Kawasaki, T. Shono, T. Hasegawa, S. Koshihara, and H. Koinuma, Appl. Phys. Lett. 78, 958 (2001).

[11] A. Tiwari, C. Jin, A. Kvit, D. Kumar, J. F. Muth, and J. Narayan, Solid State Commun. 121, 371 (2002).

[12] S. W. Jung, S. J. An, G. Yi, C. U. Jung, S. Lee, and S. Cho, Appl. Phys. Lett. 80, 4561 (2002).

[13] D. P. Norton, S. J. Pearton, A. F. Hebard, N. Theodoropoulou, L. A. Boatner, and R. G. Wilson, Appl. Phys. Lett. 82, 239 (2003).

[14] K. R. Kittilstved, N. S. Norberg, and D. R. Gamlin, Phys. Rev. Lett. 94, 147209 (2005).

[15] M. Diaconua, H. Schmidta, H. Hochmutha, M. Lorenza, G. Benndorfa, D. Spemanna, A. Setzera, P. Esquinazia, A. Poppla, H. von Wencksterna, K.-W. Nielsenb, R. Grossb, H. Schmidc, W. Maderc, G. Wagnerd, and M. Grundmanna, J. Magn. Magn. Mater. 307, 212 (2006).

[16] P. Sharma, A. Gupta, K. V. Rao, F. J. Owens, R. Sharma, R. Ahuja, J. M. Osorio Guillen, B. Johansson, and G. A. Gehring, Nature Mater. 2, 673 (2003).

[17] M. Diaconu, H. Schmidt, H. Hochmuth, M. Lorenz, G. Benndorf, J. Lenzner, D. Spemann, Setzer, K.-W. Nielsen, P. Esquinazi, and M. Grundmann, Thin Solid Films 486, 117 (2005).

[18] T. Dietl, H. Ohno, F. Matsukura, J. Cibert, and D. Ferrand, Science 287, 1019 (2000).

[19] F. Gu, S. F. Wang, M. K. Lu, G. J. Zhou, D. Xu, and D. R. Yuan, Langmuir 20, 3528 (2004).

[20] D. Kundaliya, S. Ogale, S. Lofland, S. Dhar, C. Metting, S. Shinde, Z. Ma, B. Varughese, K. Ramanujachary, L. Salamanca-Riba, and T. Venkatesan, Nature Mater. 3, 709 (2004).

[21] M. H. Ham, S. Yoon, Y. Park, and J. M. Myoung, Appl. Surf. Sci. 252, 6289 (2006).

[22] Li Zeng, A. Huege, E. Helgren, F. Hellman, C. Piamonteze, and E. Arenholz, App. Phys. Lett. 92, 142503 (2008).

[23] Y. Q. Chang, D. B. Wang, X. H. Luo, X. Y. Xu, X. H. Chen, C. P. Chen, R. M. Wang, J. Xu, and D. P. Yu, Appl. Phys. Lett. 83, 4020 (2003).

[24] L. Ying-Bin, L. Zhi-Hai, Z. Wen-Qin, L. Zhong-Lin, X. Jiang-Ping, J. Jian-Ti, L. Xing-Chong, W. Jian-Feng, L. Li-ya, Z. Feng-Ming, D. You-Wei, H. Zhi-Guo, and Z. Jian-Guo, Chinese Phys. Lett. 24, 2085 (2007).

[25] S. J. Pearton, W. H. Heo, M. Ivill, D. P. Norton, and T. Steiner, Semicond. Sci. Technol. 19, 59 (2004). 